\title{
Lie triple ideals and Lie triple epimorphisms on Jordan and Jordan-Banach algebras
}

\author{
by \\ M. BrešAr (Maribor), M. Cabrera (Granada), M. Fošner (Ljubljana) \\ and A. R. Villena (Granada)
}

\begin{abstract}
A linear subspace $M$ of a Jordan algebra $J$ is said to be a Lie triple ideal of $J$ if $[M, J, J] \subseteq M$, where $[\cdot, \cdot, \cdot]$ denotes the associator. We show that every Lie triple ideal $M$ of a nondegenerate Jordan algebra $J$ is either contained in the center of $J$ or contains the nonzero Lie triple ideal $[U, J, J]$, where $U$ is the ideal of $J$ generated by $[M, M, M]$.

Let $H$ be a Jordan algebra, let $J$ be a prime nondegenerate Jordan algebra with extended centroid $C$ and unital central closure $\widehat{J}$, and let $\Phi: H \rightarrow J$ be a Lie triple epimorphism (i.e. a linear surjection preserving associators). Assume that $\operatorname{deg}(J) \geq 12$. Then we show that there exist a homomorphism $\Psi: H \rightarrow \widehat{J}$ and a linear map $\tau: H \rightarrow C$ satisfying $\tau([H, H, H])=0$ such that either $\Phi=\Psi+\tau$ or $\Phi=-\Psi+\tau$.

Using the preceding results we show that the separating space of a Lie triple epimorphism between Jordan-Banach algebras $H$ and $J$ lies in the center modulo the radical of $J$.
\end{abstract}

1. Introduction. An associative algebra $A$ becomes a Lie algebra $A^{-}$ under the commutator

$$
[x, y]=x y-y x,
$$

and a linear Jordan algebra $A^{+}$under the Jordan product

$$
x \circ y=\frac{1}{2}(x y+y x) .
$$

In the case $A$ has a linear involution $*$, the set of skew elements

$$
K(A, *)=\left\{x \in A: x^{*}=-x\right\}
$$

is a subalgebra of $A^{-}$, and the set of symmetric elements

$$
H(A, *)=\left\{x \in A: x^{*}=x\right\}
$$

is a subalgebra of $A^{+}$. I. N. Herstein initiated the study of the ideals of $A^{-}$, $A^{+}, K(A, *)$, and $H(A, *)$ in the context of simple algebras. His work has

2000 Mathematics Subject Classification: 17C10, 17C65, 46H40, 46H70.

The first named author is supported by MŠZŠ grant P0-0501-0101.

The second named author is supported by Junta de Andalucia grant FQM290.

The fourth named author is supported by MCYT grant BFM2003-01681.

A preliminary version of this paper was received by the Editors on April 18, 2003. 
been generalized in various directions by Herstein himself and many other authors (see $[25,26,27]$ ). For a complete account of Herstein's Lie theory in the prime context, especially from the point of view of the GPI theory, we refer to [31]. Concerning Herstein's Jordan theory the reader is referred to [32] for Jordan algebras and to [1] for Jordan systems.

One of the basic results of Herstein's Lie theory is the following well known theorem (see [25, pp. 4-5]).

Herstein's TheOREm. Let $A$ be a semiprime associative algebra, let $L$ be a Lie ideal of $A$, and let $U$ be the ideal of $A$ generated by $[L, L]$. Then either $L \subseteq Z(A)$, the center of $A$, or $0 \neq[U, A] \subseteq L$.

At his 1961 AMS Hour Talk [24], I. N. Herstein posed, as Problem 2, the problem of determination of Lie isomorphisms of simple associative algebras. This problem was studied mainly by W. S. Martindale and his students, while the final breakthrough was made by M. Brešar [15] who obtained the description of Lie isomorphisms of prime associative algebras not satisfying the standard polynomial identity $\mathrm{St}_{4}$. This result was recently generalized by K. I. Beidar and M. A. Chebotar in [9] as follows.

Beidar-Chebotar Theorem. Let $A$ be an associative algebra, let $B$ be a prime associative algebra with extended centroid $C$ and unital central closure $\widehat{B}$, and let $\Phi: A \rightarrow B$ be a Lie epimorphism. Suppose that $B$ does not satisfy $\mathrm{St}_{6}$. Then there exist linear maps $\Psi: A \rightarrow \widehat{B}$ and $\tau: A \rightarrow C$ such that:

(a) $\tau([A, A])=0$, and

(b) either $\Psi$ is a homomorphism and $\Phi=\Psi+\tau$, or $\Psi$ is an antihomomorphism and $\Phi=-\Psi+\tau$.

A particularly important notion in the automatic continuity theory is that of the separating space $\mathfrak{S}(\Phi)$ of a linear map $\Phi$ from a Banach space $X$ into a Banach space $Y$, which is defined as follows:

$$
\mathfrak{S}(\Phi)=\left\{y \in Y \text { : there exists } x_{n} \rightarrow 0 \text { in } X \text { with } \Phi\left(x_{n}\right) \rightarrow y\right\} .
$$

The separating space is a closed subspace of $Y$. It is an immediate restatement of the closed graph theorem that $\Phi$ is continuous if and only if $\mathfrak{S}(\Phi)=\{0\}$.

Using the above Herstein theorem and Brešar's description of Lie isomorphisms, in [12] M. I. Berenguer and A. R. Villena obtained an automatic continuity result for Lie isomorphisms between semisimple Banach algebras. With different techniques, this result was extended by B. Aupetit and M. Mathieu in [4] who obtained the following optimal result on the continuity of Lie homomorphisms. 
Aupetit-Mathieu Theorem. Let $A$ and $B$ be Banach algebras and let $\Phi: A \rightarrow B$ be a Lie epimorphism. Then the separating space $\mathfrak{S}(\Phi)$ is contained in $\mathcal{Z}(B)$, the center modulo the radical of $B$.

If $J$ is a Jordan algebra with product denoted by $\circ$, then $J$ gets the Lie triple structure via the associator

$$
[x, y, z]=(x \circ y) \circ z-x \circ(y \circ z) .
$$

A linear subspace $M$ of $J$ will be called a Lie triple ideal of $J$ if

$$
[M, J, J] \subseteq M \text {. }
$$

Lie triple ideals are nothing other than the so-called associator ideals of [29]. A linear map $\Phi$ from a Jordan algebra $H$ onto $J$ is called a Lie triple homomorphism if

$$
\Phi([x, y, z])=[\Phi(x), \Phi(y), \Phi(z)] \quad \text { for all } x, y, z \in H .
$$

The concept of a Lie triple ideal of a Jordan algebra can be viewed as a generalization of the concept of a Lie ideal of an associative algebra. Namely, if $L$ is a Lie ideal of an associative algebra $A$, then $L$ is a Lie triple ideal of the special Jordan algebra $A^{+}$. Analogously, the concept of a Lie triple homomorphism between Jordan algebras generalizes the concept of a Lie homomorphism between associative algebras. We refer the reader to [29, Chapter VIII] for an introduction to the Lie triple structure in Jordan algebras.

The original motivation for this paper was the question of automatic continuity of Lie triple epimorphisms in Jordan-Banach algebras. In order to solve this question we obtained a description of Lie triple epimorphisms onto prime nondegenerate Jordan algebras whose degree is large enough; in this context we mention the related recent paper [18] describing Lie triple isomorphisms between centrally closed prime nondegenerate Jordan algebras. On the other hand, in order to deal with algebras of small degrees, we have needed an appropriate version of Herstein's theorem. The goal of this paper is to prove the following three results which are the Jordan algebra analogues of the aforementioned theorems by Herstein, Beidar-Chebotar, and Aupetit-Mathieu.

THEOREM 1.1. Let $M$ be a Lie triple ideal of a nondegenerate Jordan algebra $J$ and let $U$ be the ideal of $J$ generated by $[M, M, M]$. Then either $M \subseteq Z(J)$, the center of $J$, or $0 \neq[U, J, J] \subseteq M$.

THEOREM 1.2. Let $H$ be a Jordan algebra, let $J$ be a prime nondegenerate Jordan algebra with extended centroid $C$ and unital central closure $\widehat{J}$, and let $\Phi: H \rightarrow J$ be a Lie triple epimorphism. Assume that $\operatorname{deg}(J) \geq 12$. Then there exist a homomorphism $\Psi: H \rightarrow \widehat{J}$ and a linear map $\tau: H \rightarrow C$ satisfying $\tau([H, H, H])=0$ such that either $\Phi=\Psi+\tau$ or $\Phi=-\Psi+\tau$. 
THeOREm 1.3. Let $H$ and $J$ be Jordan-Banach algebras and let $\Phi: H \rightarrow$ $J$ be a Lie triple epimorphism. Then $\mathfrak{S}(\Phi) \subseteq \mathcal{Z}(J)$, the center modulo the radical of $J$.

Our proofs strongly rely on Zelmanov's structure theory for Jordan algebras, and on the recently developed theory of functional identities. In Section 2 we shall recall several definitions and briefly survey all tools of the general theory of Jordan algebras that we need. Section 3 is devoted to Lie triple ideals and its goal is to establish Theorem 1.1, which is obtained via Zelmanov's structure theory and some results on commuting maps [14, 30]. In Section 4 we outline the theory of K. I. Beidar and M. A. Chebotar on $d$-free sets $[7,8,9]$ and prove Theorem 1.2. Finally, Section 5 is devoted to the study of the separating space of Lie triple epimorphisms and its goal is to establish Theorem 1.3. The proof depends upon Theorems 1.1 and 1.2, as well as upon the result by B. Aupetit [3] on the automatic continuity of epimorphisms in Jordan-Banach algebras.

2. Preliminaries. Throughout the paper we assume that all algebras considered (associative or nonassociative) are over a field $\mathbb{F}$ of characteristic not 2 . In the analytic setting, i.e. when treating algebras that are also Banach spaces, we shall assume that $\mathbb{F}=\mathbb{C}$. These conventions will be used without further mention.

Let $A$ be an arbitrary (not necessarily associative or unital) algebra. Recall that the center $Z$ of $A$ consists of elements $c \in A$ such that $[c, A]=$ $[c, A, A]=[A, c, A]=[A, A, c]=0$ (note that in the case when $A$ is commutative it suffices to verify that at least one of the identities $[c, A, A]=0$, $[A, c, A]=0$, and $[A, A, c]=0$ is satisfied). If $A$ has a unity $\mathbf{1}$, then $A$ is called central over $\mathbb{F}$ if $Z=\mathbb{F} \mathbf{1}$. The algebra $A$ is said to be prime if the product of any two of its nonzero ideals is nonzero. Recall that if $A$ is a prime algebra with a nonzero center $Z$, then $A$ has no zero divisors in $Z$, and as a consequence one can consider the central localization $(Z \backslash\{0\})^{-1} A$, which is a central prime algebra over $(Z \backslash\{0\})^{-1} Z$ (the field of fractions of $Z$ ) [41, p. 185]. In the case that $(Z \backslash\{0\})^{-1} A$ is simple, $(Z \backslash\{0\})^{-1} A$ is the central closure of $A$ and $(Z \backslash\{0\})^{-1} Z$ is the extended centroid of $A$ [17, Lemma 3.2]. The reader is referred to [22] (resp. [6]) for a more detailed account of these concepts in the prime (resp. semiprime) context. We recall that, for $A$ prime, the extended centroid $C=C(A)$ of $A$ can be seen as a field extension of the base field $\mathbb{F}$. The algebra $A$ is said to be centrally closed (over $\mathbb{F}$ ) if the canonical embedding of $\mathbb{F}$ into $C$ is surjective. The central closure of $A$ is a centrally closed prime algebra over $C$ which can be regarded as an extension algebra of $A$ and it is generated by $A$ as an algebra over $C$. The unital central closure $\widehat{A}$ of $A$ is defined as the unital envelope of the central closure of $A$. We remark that the extended centroid $C$ is the center of $\widehat{A}$. 
In the associative context, the extended centroid and the central closure can be viewed inside the symmetric Martindale algebra of quotients. Recall that the symmetric Martindale algebra of quotients of a prime associative algebra $A$, denoted here by $Q(A)$, can be introduced as the associative algebra which is the maximal extension $Q$ of $A$ satisfying the following conditions:

(i) for each $q$ in $Q$ there exists a nonzero ideal $U$ of $A$ such that

$$
q U+U q \subseteq A ;
$$

(ii) if $q$ in $Q$ satisfies $q U=0$ for some nonzero ideal $U$ of $A$, then $q=0$. It is well known that the extended centroid $C(A)$ is the center of $Q(A)$ and the unital central closure $\widehat{A}$ is the unital $C(A)$-subalgebra of $Q(A)$ generated by $A$ (see $[10])$.

A Jordan algebra is a nonassociative algebra $J$ whose product $\circ$ satisfies $x \circ y=y \circ x$ and $\left(x^{2} \circ y\right) \circ x=x^{2} \circ(y \circ x)$ for all $x, y \in J$. In other words, $J$ is commutative and $\left[x^{2}, y, x\right]=0$ for all $x, y \in J$. For every $a \in J$ we define an operator $U_{a}: J \rightarrow J$ by

$$
U_{a}(x)=2 a \circ(a \circ x)-a^{2} \circ x .
$$

We say that $J$ is a nondegenerate Jordan algebra if $U_{a}=0$ with $a \in J$ implies $a=0$.

A Jordan algebra $J$ is said to be special if it can be embedded into $A^{+}$ for some associative algebra $A$. Besides $A^{+}$itself and $H(A, *)$ in the case when $A$ is endowed with a linear involution $*$, another standard example of a special Jordan algebra is the Jordan algebra $J(X, f)$ of a symmetric bilinear form $f$ on a linear space $X$ over $\mathbb{F}: J(X, f)=\mathbb{F} \oplus X$ with the product given by $(\alpha+x) \circ(\beta+y)=\alpha \beta+f(x, y)+\beta x+\alpha y$ for all $\alpha, \beta \in \mathbb{F}$ and $x, y \in X$. The algebra $J(X, f)$ is quadratic and in fact every quadratic Jordan algebra is of this form. Moreover, if $\operatorname{dim} X \geq 2$, then $J(X, f)$ is a central simple Jordan algebra if and only if $f$ is nondegenerate.

A Jordan algebra that is not special is called exceptional. Examples of exceptional Jordan algebras come from Albert algebras $H_{3}(D, \Gamma)$ : the set of all $3 \times 3$-matrices with entries in the Cayley-Dickson algebra $D$ which are hermitian under the involution $X \mapsto X^{*}=\Gamma^{-1} \bar{X}^{t} \Gamma$ (where $\Gamma$ stands for the diagonal matrix $\operatorname{diag}\left\{\gamma_{1}, \gamma_{2}, \gamma_{3}\right\}$ with $\gamma_{1}, \gamma_{2}, \gamma_{3}$ nonzero elements in $\mathbb{F}$ and $\bar{X}^{t}$ is given by transposing the matrix $X$ and taking the Cayley involution on each entry) endowed with the symmetrized product of the usual matrix product is a central simple 27-dimensional Jordan algebra. In the case when the base field $\mathbb{F}$ is algebraically closed there is a unique Cayley-Dickson algebra $\mathbb{O}$ (the so-called octonions over $\mathbb{F}$ ) and the only finite-dimensional exceptional simple Jordan algebra is $H_{3}(\mathbb{O})\left(=H_{3}(\mathbb{O}, \Gamma)\right.$, where $\Gamma$ is equal to the identity matrix). 
In his celebrated work [39] Zelmanov proved the following classification theorem.

Zelmanov's Prime Theorem. A Jordan algebra $J$ is prime nondegenerate if and only if one of the following possibilities holds:

(1) $J$ is a central order in an Albert algebra over a field extension of $\mathbb{F}$;

(2) $J$ is a central order in the Jordan algebra $J(X, f)$ of a nondegenerate symmetric bilinear form over a field extension of $\mathbb{F}$;

(3) there exists a prime associative algebra $A$ over $\mathbb{F}$ such that $J$ can be embedded as a Jordan subalgebra into $Q(A)$ and $A$ is an ideal of $J$;

(4) there exists a prime associative algebra $A$ over $\mathbb{F}$ with involution $*$ such that $J$ can be embedded into $H(Q(A), *)$ and $H(A, *)$ is an ideal of $J$.

Let $A$ be a prime power associative algebra with extended centroid $C$. An element $x \in A$ is said to be algebraic over $C$ of degree $\leq n$ if there exist $c_{0}, c_{1}, \ldots, c_{n} \in C$ (some of them different from zero) such that $\sum_{i=0}^{n} c_{i} x^{i}=0$. An element $x \in A$ is said to be algebraic over $C$ of degree $n$ if it is algebraic over $C$ of degree $\leq n$ and is not algebraic over $C$ of degree $\leq n-1$. By $\operatorname{deg}_{A}(x)$ we shall mean the degree of $x$ (if $x$ is algebraic over $C$ ) or $\infty$ (if $x$ is not algebraic over $C$ ). Given a nonempty subset $R \subseteq A$, we set

$$
\operatorname{deg}_{A}(R)=\sup \left\{\operatorname{deg}_{A}(x): x \in R\right\} .
$$

We write $\operatorname{deg}(A)$ instead of $\operatorname{deg}_{A}(A)$. If $A$ is a prime associative algebra with $\operatorname{deg}(A)=n<\infty$, then it follows from the PI-theory that $A$ is isomorphic to a subalgebra $B$ of $M_{n}(\bar{C})(\bar{C}$ is the algebraic closure of $C)$ such that $\bar{C} B=M_{n}(\bar{C})$.

The standard concept of invertibility in associative algebras was extended to the context of Jordan algebras by N. Jacobson. We say that an element $x$ in a unital Jordan algebra $J$ is invertible if there exists $y \in J$ such that $x \circ y=\mathbf{1}$ and $x^{2} \circ y=x$. Further, an element $x$ in a possibly nonunital Jordan algebra $J$ is quasi-invertible if $\mathbf{1}-x$ is invertible in its unital envelope $J^{1}$. K. McCrimmon [33] proved that in each Jordan algebra $J$ there exists the largest ideal consisting of quasi-invertible elements. This ideal is called the Jacobson-McCrimmon radical of $J$ and denoted by $\operatorname{Rad}(J)$. We say that $J$ is semisimple if $\operatorname{Rad}(J)=0$. If $A$ is an associative algebra, then $\operatorname{Rad}\left(A^{+}\right)$coincides with the classical Jacobson radical of $A$ (see [33]). We define the center modulo the radical of the Jordan algebra $J$ by

$$
\mathcal{Z}(J)=\{z \in J:[z, J, J] \subseteq \operatorname{Rad}(J)\} .
$$

E. I. Zelmanov [38] introduced the notion of primitiveness for unital Jordan algebras to derive his classification of prime nondegenerate Jordan algebras. This concept was extended to nonunital Jordan algebras by L. Hogben and 
K. McCrimmon [28]. A linear subspace $I$ of $J$ is said to be an inner ideal of $J$ if $U_{I}\left(J^{1}\right) \subseteq I$. We call an ideal $P$ of $J$ primitive if it is the largest ideal of $J$ contained in a maximal-modular inner ideal of $J$ (see [28] for the definition of modularity). It turns out that $\operatorname{Rad}(J)$ is the intersection of all primitive ideals of $J$ and that the classical primitive ideals of an associative algebra $A$ are primitive ideals of the Jordan algebra $A^{+}$(see [28]). A Jordan algebra $J$ is said to be primitive if 0 is a primitive ideal of $J$. Primitive Jordan algebras are prime nondegenerate. A zelmanovian description of primitive Jordan algebras was obtained independently by J. A. Anquela, F. Montaner, and T. Cortés [2], and by V. G. Skosyrskiŭ [36]. A structure theorem for primitive Jordan-Banach algebras was obtained by M. Cabrera, A. Moreno, and A. Rodríguez in [20] (see also [19]).

By a Jordan-Banach algebra we mean a complex Jordan algebra $J$ whose underlying vector space is a Banach space with respect to the norm $\|\cdot\|$ satisfying $\|x \circ y\| \leq\|x\|\|y\|$ for all $x, y \in J$. For example, if $A$ is an (associative) Banach algebra, then $A^{+}$is a Jordan-Banach algebra.

3. Herstein's theorem for Lie triple ideals. We begin with some general comments on Lie triple ideals. Let $J$ be a Jordan algebra. Every Lie triple ideal $M$ of $J$ automatically satisfies $[J, J, M] \subseteq M$ and $[J, M, J] \subseteq M$. Ideals of $J$ are of course also Lie triple ideals of $J$. There are other examples: the center $Z(J)$ of $J$ is a Lie triple ideal of $J$, and so is any subspace of $Z(J)$. If $M$ is a Lie triple ideal of $J$ then $[M, J, J]$ is a Lie triple ideal of $J$.

Lemma 3.1. Let $J$ be a Jordan algebra. If $M$ is a Lie triple ideal of $J$ and $U$ is the ideal of $J$ generated by $[M, M, M]$, then $[U, J, J] \subseteq M$.

Proof. First note that $[M, M, M]$ is a subspace of $J$ which is invariant under the inner derivations of $J$ and is contained in $M$. Therefore, by [39, identity (3), p. 73] (or [34, (12.11)]), we have

$$
U=[M, M, M]+J \circ[M, M, M]+J \circ(J \circ[M, M, M]) .
$$

Since, for $a, x, y, z \in J$,

$$
a \circ[x, y, z]+[a, x, y] \circ z=[a \circ x, y, z]-[a, x \circ y, z]+[a, x, y \circ z]
$$

(see [29, identity (30), p. 18]), it follows that $J \circ[M, M, M] \subseteq M+M \circ M$. From this and the obvious equality

$$
a \circ(b \circ[x, y, z])=(a \circ b) \circ[x, y, z]-[a, b,[x, y, z]]
$$

we deduce that $J \circ(J \circ[M, M, M]) \subseteq M+M \circ M$. Therefore $U \subseteq M+M \circ M$. Finally, using the linearization of the Jordan identity

$$
[x \circ y, a, b]+[b \circ y, a, x]+[b \circ x, a, y]=0,
$$

it follows that $[M \circ M, J, J] \subseteq M$. Thus, we see that $[U, J, J] \subseteq M$. 
We shall examine Lie triple ideals of basic examples of Jordan algebras $J$ which appear in the classification theorem. Our purpose is to prove case by case that for a Lie triple ideal $M$ of $J$ the property $[M, M, M]=0$ entails $M \subseteq Z(J)$.

3.1. Case (1). Let $H_{3}(\mathbb{O})$ be the unique Albert algebra over a given algebraically closed field. In order to avoid the matrix notation, we set

$$
\lambda \mathcal{E}_{11}=\left(\begin{array}{ccc}
\lambda & 0 & 0 \\
0 & 0 & 0 \\
0 & 0 & 0
\end{array}\right), \quad x \mathcal{E}_{12}=\left(\begin{array}{ccc}
0 & x & 0 \\
\bar{x} & 0 & 0 \\
0 & 0 & 0
\end{array}\right), \quad \text { etc. }
$$

Therefore, every element in $J$ can be written as

$$
A=\lambda \mathcal{E}_{11}+\mu \mathcal{E}_{22}+\nu \mathcal{E}_{33}+x \mathcal{E}_{12}+y \mathcal{E}_{13}+z \mathcal{E}_{23} .
$$

Note that

$$
4\left[A, \mathcal{E}_{11}, \mathcal{E}_{22}\right]=x \mathcal{E}_{12}, \quad 4\left[A, \mathcal{E}_{11}, \mathcal{E}_{33}\right]=y \mathcal{E}_{13}, \quad 4\left[A, \mathcal{E}_{22}, \mathcal{E}_{33}\right]=z \mathcal{E}_{23} .
$$

Let $J^{\prime}$ stand for the set of all elements in $H_{3}(\mathbb{O})$ with trace zero. We now claim that $J^{\prime}=[J, J, J]$. Indeed, from (1) we see that every matrix in $J$ with zero diagonal lies in $[J, J, J]$, and so it follows from

$$
\mathcal{E}_{i i}-\mathcal{E}_{j j}=-2\left[\mathcal{E}_{i i}, \mathcal{E}_{i j}, \mathcal{E}_{i j}\right] \quad(1 \leq i<j \leq 3)
$$

that every matrix with trace zero lies in $[J, J, J]$. The converse is a direct consequence of the fact that the trace of an Albert algebra is associative [29, Corollary 4, p. 227].

Finally it should be noted that $J^{\prime}=\left[J^{\prime}, J^{\prime}, J^{\prime}\right]$. This is a consequence of the identities

$$
\left[\mathcal{E}_{i i}-\mathcal{E}_{j j}, \mathcal{E}_{i i}-\mathcal{E}_{j j}, x \mathcal{E}_{i j}\right]=x \mathcal{E}_{i j} \quad(1 \leq i<j \leq 3)
$$

and

$$
\left[\mathcal{E}_{i j}, \mathcal{E}_{i j}, \mathcal{E}_{i i}-\mathcal{E}_{j j}\right]=\mathcal{E}_{i i}-\mathcal{E}_{j j} \quad(1 \leq i<j \leq 3) .
$$

Proposition 3.2. Let $J$ be a prime nondegenerate exceptional Jordan algebra. If $M$ is a Lie triple ideal of $J$ such that $[M, M, M]=0$, then $M \subseteq Z(J)$.

Proof. Let $\widehat{J}$ denote the central localization of $J$ and let $\mathbb{K}$ be the algebraic closure of the field of fractions of $Z(J)$. Consider the scalar extension $\widetilde{J}$ of $\widehat{J}$ over $\mathbb{K}$. If $M$ is a Lie triple ideal of $J$ such that $[M, M, M]=0$, then $\widetilde{M}:=\mathbb{K} M$ is clearly a Lie triple ideal of $\widetilde{J}$ such that $[\widetilde{M}, \widetilde{M}, \widetilde{M}]=0$. By $[29$, Theorem 17, p. 408], the Lie triple ideals of $\widetilde{J}$ are: $0, \mathbb{K} \mathbf{1}, \widetilde{J}^{\prime}$, and $\widetilde{J}$. Since $[\widetilde{J}, \widetilde{J}, \widetilde{J}]=\left[\widetilde{J}^{\prime}, \widetilde{J}^{\prime}, \widetilde{J}^{\prime}\right]=\widetilde{J}^{\prime}$, it follows that $\widetilde{M}=0$ or $\widetilde{M}=\mathbb{K} \mathbf{1}$. Therefore, $\widetilde{M} \subseteq Z(\widetilde{J})$, and so $M \subseteq Z(J)$ 
3.2. Case (2). We continue by treating Jordan algebras which are central orders in the Jordan algebra $J(X, f)$ of a nondegenerate symmetric bilinear form over a field extension of $\mathbb{F}$.

Lemma 3.3. Let $X$ be a vector space over $\mathbb{F}$ with $\operatorname{dim} X \geq 2$, let $f$ be a nondegenerate symmetric bilinear form on $X$, and let $J=J(X, f)$. Then, for every pair of linearly independent elements $x, z$ in $X$, there exists $y \in X$ such that $[x, y, z]=z$. As a consequence, $[J, J, J]=[X, X, X]=X$, and the Lie triple ideals of $J$ are: $0, \mathbb{F} \mathbf{1}, X$, and $J$.

Proof. Since $x$ and $z$ are linearly independent, there is $y \in X$ such that $f(z, y)=0$ and $f(x, y)=1$; accordingly, $[x, y, z]=z$. Using this, one easily infers that $[J, J, J]=[X, X, X]=X$. Now let $M$ be a Lie triple ideal of $J$ different from 0 and $\mathbb{F} \mathbf{1}(=Z(J))$. Pick $\lambda+x \in M$ such that $x \neq 0$. If $z \in X$ is such that $x$ and $z$ are linearly independent, then, as noted above, there is $y \in X$ such that $[\lambda+x, y, z]=z$. Thus $M$ contains all vectors in $X$ that are linearly independent of $x$. However, since $x=z+(x-z), M$ contains $x$ as well. Therefore $[J, J, J]=X \subseteq M$ and so either $M=[J, J, J]$ or $M=J$.

Proposition 3.4. Let $J$ be a Jordan algebra which is a central order in a central simple quadratic Jordan algebra. If $M$ is a Lie triple ideal of $J$ such that $[M, M, M]=0$, then $M \subseteq Z(J)$.

Proof. If $J$ is associative, then the statement is clear. Assume that $J$ is not associative, and denote by $\mathbb{K}$ the field of fractions of $Z(J)$ and by $\widehat{J}$ the central localization of $J$. Then $\widehat{J}=J(X, f)$ for a suitable vector space $X$ over $\mathbb{K}$ with $\operatorname{dim} X \geq 2$ and a nondegenerate symmetric bilinear form $f$ on $X$. If $M$ is a Lie triple ideal of $J$ such that $[M, M, M]=0$, then $\widehat{M}:=\mathbb{K} M$ is a Lie triple ideal of $\widehat{J}$ such that $[\widehat{M}, \widehat{M}, \widehat{M}]=0$. By the above lemma, we have $\widehat{M} \subseteq Z(\widehat{J})$, and so $M \subseteq Z(J)$

3.3. Case (3). Now we assume that $J$ is as in (3) of Zelmanov's theorem, i.e. there is a prime associative algebra $A$ such that $J$ is a Jordan subalgebra of $Q(A)$ containing $A$ as an ideal. Note that in $Q(A)$ we have

$$
4[x, y, z]=[y,[x, z]]
$$

for all $x, y, z$ in $J$, where $[\cdot, \cdot, \cdot]$ stands for the associator on $J$ and $[\cdot, \cdot]$ stands for the commutator on $Q(A)$. We now record a straightforward generalization of [25, Sublemma, p. 5].

LEMMA 3.5. Let $A$ be a semiprime associative algebra. If $q \in Q(A)$ is such that $[q,[q, A]]=0$, then $q \in C(A)$.

Proposition 3.6. Let $A$ be a prime associative algebra, and let $J$ be a Jordan subalgebra of $Q(A)$ containing $A$ as an ideal. If $M$ is a Lie triple ideal of $J$ such that $[M, M, M]=0$, then $M \subseteq Z(J)$. 
Proof. Let $M$ be a Lie triple ideal of $J$ such that $[M, M, M]=0$. We begin by noting that for each $x, y \in M$ and $a \in A$ we see that $[[x, y], a]$ belongs to $M$, so $[[x, y],[[x, y], a]]=0$, and so, by Lemma $3.5,[x, y] \in C(A)$. Thus $[M, M] \subseteq C(A)$. We claim that in fact $[M, M]=0$. Indeed, for $x, y$ $\in M$, both $y x y=U_{y}(x)$ and $y x^{2} y=U_{y}\left(x^{2}\right)$ belong to $J$, so $[x,[x, y x y]]$ and $\left[y,\left[y, y x^{2} y\right]\right]$ belong to $M$, and so

$$
\left[x,[x,[x, y x y]],\left[y,\left[y, y x^{2} y\right]\right]\right]=0 .
$$

Since $[x,[x, y x y]]=2[x, y]^{2} x$ and $\left[y,\left[y, y x^{2} y\right]\right]=2[x, y]^{2} y^{2}$, it follows that

$$
0=\left[x,[x, y]^{2} x,[x, y]^{2} y^{2}\right]=\frac{1}{2}[x, y]^{6},
$$

and consequently $[x, y]=0$ because $[x, y] \in C(A)$ and $C(A)$ is a field, which proves the claim.

For $x \in M$ and $a \in A$ we have

$$
[[x,[x, a]], a]=[[x, a],[x, a]]+[x,[[x, a], a]]=0 .
$$

Since $[x,[x, a]]=4[x, x, a] \in A$, we can apply $[14$, Theorem $\mathrm{A}]$, giving the existence of $\lambda_{x} \in C(A)$ and a linear mapping $\zeta_{x}: A \rightarrow C(A)$ such that $[x,[x, a]]=\lambda_{x} a+\zeta_{x}(a)$. Since $[M, M]=0$ we have $[x,[x,[x, a]]]=0$, which yields $\lambda_{x}[x, a]=0$. Thus either $\lambda_{x}=0$ or $[x, a]=0$. In any case, $[x,[x, a]] \in$ $C(A)$ for all $x \in M$ and $a \in A$. In particular, we have $[x,[x, a \circ x]] \in C(A)$ because $a \circ x \in A$. Hence we deduce that

$$
\begin{aligned}
{[x,[x, a]]^{2} } & =[[x,[x, a]] x,[x, a]]=[[x,[x, a]] \circ x,[x, a]] \\
& =[[x,[x, a] \circ x],[x, a]]=[[x,[x, a \circ x]],[x, a]]=0,
\end{aligned}
$$

and hence $[x,[x, a]]=0$. Therefore, applying Lemma 3.5 again, we obtain $x \in C(A)$. Thus $M \subseteq C(A)$. Finally, from the obvious fact that $C(A) \cap J \subseteq$ $Z(J)$, we conclude that $M \subseteq Z(J)$.

3.4. Case (4). Now we assume that $A$ is a prime associative algebra with involution $*$ and $J$ is a subalgebra of $H(Q(A), *)$ containing $H(A, *)$ as an ideal. We continue by considering associators in $J$ as the corresponding double commutators on $Q(A)$.

Lemma 3.7. Let $A$ be a semiprime associative algebra with involution $*$. If $q \in H(Q(A), *)$ is such that $[q,[q, H(A, *)]]=0$, then $q \in C(A)$.

Proof. Using [10, Theorem 6.4.7] we see that $[q,[q, H(A, *)]]=0$ implies $[q,[q, H(Q(A), *)]]=0$. Applying $[30$, Theorem 2], we deduce $[q,[q, Q(A)]]$ $=0$. Finally, the conclusion $q \in C(A)$ follows from Lemma 3.5.

Proposition 3.8. Let $A$ be a prime associative algebra with involution * and let $J$ be a Jordan subalgebra of $H(Q(A), *)$ containing $H(A, *)$ as an ideal. If $M$ is a Lie triple ideal of $J$ such that $[M, M, M]=0$, then $M \subseteq Z(J)$. 
Proof. Assume first that $[H(A, *), H(A, *)]=0$. By Lemma 3.7, $H(A, *)$ $\subseteq C(A)$. A second application of Lemma 3.7 yields $H(Q(A), *) \subseteq C(A)$ and so $J \subseteq C(A)$. Now $M \subseteq Z(J)$ trivially follows.

So we may assume that $[H(A, *), H(A, *)] \neq 0$. We note that, for $x, y \in$ $M$ and $h \in H(A, *)$, we have $[[x, y],[[x, y], h]]=0$ because $[[x, y], h]$ belongs to $M$. Hence $\left[[x, y]^{2},\left[[x, y]^{2}, h\right]\right]=0$, and therefore, by Lemma $3.7,[x, y]^{2} \in$ $C(A)$. Now, note that both $y x y=U_{y}(x)$ and $y x^{2} y=U_{y}\left(x^{2}\right)$ belong to $J$, so $[x,[x, y x y]]$ and $\left[y,\left[y, y x^{2} y\right]\right]$ belong to $M$, and so

$$
\left[x,[x,[x, y x y]],\left[y,\left[y, y x^{2} y\right]\right]\right]=0 .
$$

Since $[x,[x, y x y]]=2[x, y] x[x, y]$ and $\left[y,\left[y, y x^{2} y\right]\right]=2[x, y]^{2} y^{2}$, it follows that

$$
0=\left[x,[x, y] x[x, y],[x, y]^{2} y^{2}\right]=\frac{1}{2}[x, y]^{6},
$$

and consequently $[x, y]^{2}=0$ because $[x, y]^{2} \in C(A)$ and $C(A)$ is a field. Therefore, for $h \in H(A, *)$, we have

$$
0=[[x, y],[[x, y], h]]=-2[x, y] h[x, y] .
$$

Using the Jacobi identity we see that

$$
[[M, M],[H(A, *), H(A, *)]] \subseteq[[M,[H(A, *), H(A, *)]], M] \subseteq[M, M]
$$

and

$$
[[M, M],[M, M]] \subseteq[[[M, M], M], M]=[[M, M, M], M]=0,
$$

which yields

$$
[[M, M],[[M, M],[H(A, *), H(A, *)]]] \subseteq[[M, M],[M, M]]=0 .
$$

Therefore

$$
0=\left[[x, y],\left[[x, y],\left[h_{1}, h_{2}\right]\right]\right]=-2[x, y]\left[h_{1}, h_{2}\right][x, y]
$$

for all $h_{1}, h_{2} \in H(A, *)$ and $x, y \in M$. Since $[x, y] H(A, *)[x, y]=0$ as well, we now have

$$
[x, y](H(A, *)+[H(A, *), H(A, *)])[x, y]=0
$$

and so, making use of [10, Theorem 6.4.7], also

$$
[x, y](H(Q(A), *)+[H(Q(A), *), H(Q(A), *)])[x, y]=0
$$

for all $x, y \in M$. Note that $H(Q(A), *)+[H(Q(A), *), H(Q(A), *)]$ is a Lie ideal of $Q(A)$. Moreover, since $[H(A, *), H(A, *)] \neq 0$ by our assumption, it is a noncommutative Lie ideal. Now $[13$, Lemma 4$]$ implies that $[x, y]=0$ for all $x, y \in M$. Thus we have proved that $[M, M]=0$.

Now, for $x \in M$ and $h \in H(A, *)$ we have

$$
[[x,[x, h]], h]=[[x, h],[x, h]]+[x,[[x, h], h]]=0 .
$$

Since $[x,[x, h]]=4[x, x, h] \in H(A, *)$, we can apply $[30$, Theorem 3$]$ to obtain the existence of $\lambda_{x} \in C(A)$ and a linear mapping $\mu_{x}: H(A, *) \rightarrow C(A)$ such 
that $[x,[x, h]]=\lambda_{x} h+\mu_{x}(h)$ for all $h \in H(A, *)$. Finally, we argue as in the proof of Proposition 3.6 to conclude that $M \subseteq Z(J)$.

3.5. The general case. Now we are in a position to prove Theorem 1.1.

Proof of Theorem 1.1. On account of Lemma 3.1, it is enough to show that, if $[U, J, J]=0$ (equivalently, $U \subseteq Z(J)$ ), then $M \subseteq Z(J)$. Assume that $U \subseteq Z(J)$. Then clearly $[M, M, M]$ and $J \circ[M, M, M]$ are contained in $Z(J)$. Consequently, for $x, y, z \in M$ we have

$$
\begin{aligned}
U_{[x, y, z]}(a) & =2[x, y, z] \circ([x, y, z] \circ a)-[x, y, z]^{2} \circ a \\
& =[x, y, z]^{2} \circ a=[x \circ[x, y, z], y, z] \circ a=0
\end{aligned}
$$

for each $a \in J$ and hence $[x, y, z]=0$ because $J$ is nondegenerate. Thus $[M, M, M]=0$. By [40], $J$ can be seen as a subdirect product of a family of prime nondegenerate Jordan algebras $\left\{J_{\lambda}\right\}_{\lambda \in \Lambda}$. If $\pi_{\lambda}$ denotes the canonical homomorphism from $J$ onto $J_{\lambda}$, it is clear that $M_{\lambda}:=\pi_{\lambda}(M)$ is a Lie triple ideal of $J_{\lambda}$ such that $\left[M_{\lambda}, M_{\lambda}, M_{\lambda}\right]=0$. From this and the obvious fact that $Z(J)=\bigcap_{\lambda \in \Lambda} \pi_{\lambda}^{-1}\left(Z\left(J_{\lambda}\right)\right)$ it follows that there is no loss of generality in assuming that the nondegenerate Jordan algebra $J$ is in addition prime. Then the desired conclusion $M \subseteq Z(J)$ follows from Zelmanov's prime theorem and Propositions 3.2, 3.4, 3.6, and 3.8.

\section{Lie triple epimorphisms on prime nondegenerate Jordan al-} gebras of large degree. The main goal of this section is to prove Theorem 1.2. For this we need some auxiliary results. Some of them are directly taken from [7,8], while the others are suitable Jordan versions of associative results in [9]. First, however, we have to recall some basic concepts from the theory of functional identities. For an introductory account of this theory we refer the reader to [16].

We begin by defining a $d$-free subset of a unital associative algebra $Q$ (see [7]). Let $X$ be a nonempty subset of $Q$. By $X^{n}$ we denote the Cartesian product $X \times \cdots \times X$ of $n$ copies of $X$. Let $m$ be a positive integer, and let $E: X^{m-1} \rightarrow Q, p: X^{m-2} \rightarrow Q$ be arbitrary maps. In the case when $m=1$ this should be understood as that $E$ is an element in $Q$ and $p=0$. Let $1 \leq i<j \leq m$, and define $E^{i}, p^{i j}, p^{j i}: X^{m} \rightarrow Q$ by

$$
\begin{aligned}
E^{i}\left(\bar{x}_{m}\right) & =E\left(x_{1}, \ldots, x_{i-1}, x_{i+1}, \ldots, x_{m}\right), \\
p^{i j}\left(\bar{x}_{m}\right) & =p^{j i}\left(\bar{x}_{m}\right)=p\left(x_{1}, \ldots, x_{i-1}, x_{i+1}, \ldots, x_{j-1}, x_{j+1}, \ldots, x_{m}\right) ;
\end{aligned}
$$

here, $\bar{x}_{m}=\left(x_{1}, \ldots, x_{m}\right) \in X^{m}$.

Now let $I, J \subseteq\{1, \ldots, m\}$, and for each $i \in I, j \in J$, let $E_{i}, F_{j}: X^{m-1} \rightarrow$ $Q$ be arbitrary maps. Consider the functional identities

$$
\sum_{i \in I} E_{i}^{i}\left(\bar{x}_{m}\right) x_{i}+\sum_{j \in J} x_{j} F_{j}^{j}\left(\bar{x}_{m}\right)=0 \quad \text { for all } \bar{x}_{m} \in X^{m}
$$


and

$$
\sum_{i \in I} E_{i}^{i}\left(\bar{x}_{m}\right) x_{i}+\sum_{j \in J} x_{j} F_{j}^{j}\left(\bar{x}_{m}\right) \in C \quad \text { for all } \bar{x}_{m} \in X^{m}
$$

where $C$ is the center of $Q$. A natural possibility when (3) (and hence also (4)) holds is that there exist maps $p_{i j}: X^{m-2} \rightarrow Q, i \in I, j \in J$, $i \neq j, \lambda_{k}: X^{m-1} \rightarrow C, k \in I \cup J$, such that

$$
\begin{aligned}
E_{i}^{i}\left(\bar{x}_{m}\right) & =\sum_{j \in J, j \neq i} x_{j} p_{i j}^{i j}\left(\bar{x}_{m}\right)+\lambda_{i}^{i}\left(\bar{x}_{m}\right), \\
F_{j}^{j}\left(\bar{x}_{m}\right) & =-\sum_{i \in I, i \neq j} p_{i j}^{i j}\left(\bar{x}_{m}\right) x_{i}-\lambda_{j}^{j}\left(\bar{x}_{m}\right), \\
\lambda_{k} & =0 \quad \text { if } k \notin I \cap J,
\end{aligned}
$$

for all $\bar{x}_{m} \in X^{m}, i \in I, j \in J$. Indeed, one can readily check that (5) implies (3) (we remark that (5) is called a standard solution of (3) and (4)). It should be pointed out that the case when one of the sets $I$ or $J$ is empty is not excluded. The sum over the empty set of indices should be simply read as zero. This means that, for example, the only standard solution of the functional identity $\sum_{i \in I} E_{i}^{i}\left(\bar{x}_{m}\right) x_{i}=0$ is $E_{i}=0, i \in I$.

Let $d$ be a positive integer. We say that $X$ is a $d$-free subset of $Q$ if for every positive integer $m$ and all $I, J \subseteq\{1, \ldots, m\}$ the following two conditions are satisfied:

(a) If $\max \{|I|,|J|\} \leq d$, then (3) implies (5).

(b) If $\max \{|I|,|J|\} \leq d-1$, then (4) implies (5).

So, roughly speaking, a subset is $d$-free if the functional identities (3) and (4) have only standard solutions, provided that the number of variables is sufficiently small (depending on $d$ ). It turns out that some important subsets of prime rings $A$ are, under some natural restrictions, $d$-free subsets of $Q(A)$ (see $[7])$. This justifies the introduction of the concept of a $d$-free subset.

Next we recall, in a somewhat loose manner, the definition of a quasipolynomial (see [8] for details). Let $S$ be a set and let $\alpha: S \rightarrow Q$ be a map. We say that $q: S^{2} \rightarrow Q$ is a multilinear quasi-polynomial (with respect to $\alpha$ ) of degree $\leq 2$ if there exist $\lambda_{1}, \lambda_{2} \in C$ and maps $\mu_{1}, \mu_{2}: S \rightarrow C, \nu: S^{2} \rightarrow C$ such that

$$
q(x, y)=\lambda_{1} \alpha(x) \alpha(y)+\lambda_{2} \alpha(y) \alpha(x)+\mu_{1}(x) \alpha(y)+\mu_{2}(y) \alpha(x)+\nu(x, y)
$$

for all $x, y \in S$. We call $\lambda_{1}, \lambda_{2}, \mu_{1}, \mu_{2}, \nu$ the coefficients of $q$. Further, if $S$ is a vector space, $\mu_{1}, \mu_{2}$ are linear maps and $\nu$ is a bilinear map, then we say that $q$ is a multilinear quasi-polynomial of degree $\leq 2$ with multilinear coefficients. 
A multilinear quasi-polynomial of degree $m$ would, of course, involve summands such as $\lambda \alpha\left(x_{1}\right) \cdots \alpha\left(x_{m}\right), \quad \mu\left(x_{1}\right) \alpha\left(x_{2}\right) \cdots \alpha\left(x_{m}\right), \quad \nu\left(x_{1}, x_{2}\right) \alpha\left(x_{3}\right) \cdots \alpha\left(x_{m}\right), \quad$ etc.

The next theorem is a particular case of [8, Theorem 1.1] (see also [9, Theorem 2.2]).

TheOREM 4.1. Let $Q$ be a unital associative algebra with center $C$, let $R$ be a subset of $Q$, let $S$ be a set, let $\alpha: S \rightarrow R$ be a surjective map, and let $q$ be a multilinear quasi-polynomial of degree $\leq m$ such that $q\left(\bar{s}_{m}\right)=0$ for each $\bar{s}_{m} \in S^{m}$. If $R$ is an $(m+1)$-free subset of $Q$, then all coefficients of $q$ are 0 .

The following theorem is a particular case of [8, Theorem 1.2].

TheOREM 4.2. Let $Q$ be a unital associative algebra with center $C$, let $R$ be a subspace of $Q$, let $S$ be a vector space, let $\alpha: S \rightarrow R$ be a surjective linear map, and let $B: S^{2} \rightarrow Q$ be a bilinear map such that

$$
\begin{aligned}
{\left[B\left(s_{1}, s_{2}\right), \alpha\left(s_{3}\right), \alpha\left(s_{4}\right)\right]+\left[B\left(s_{4}, s_{1}\right), \alpha\left(s_{3}\right), \alpha\left(s_{2}\right)\right] } & \\
+ & {\left[B\left(s_{2}, s_{4}\right), \alpha\left(s_{3}\right), \alpha\left(s_{1}\right)\right] \in C }
\end{aligned}
$$

for each $\bar{s}_{4} \in S^{4}$. If $R$ is a 5 -free subset of $Q$, then $B$ is a quasi-polynomial of degree $\leq 2$ with multilinear coefficients.

The following two facts will be applied throughout this section often without notice. They follow easily from [5, Section 3] and [6, Section 3].

THEOREM 4.3. Let $A$ be a prime associative algebra and let $J$ be a Jordan subalgebra of $Q(A)$ containing $A$ as an ideal. Then there is an isomorphism from $C(J)$ onto $C(A)$ that extends to an isomorphism from the unital central closure of $J$ onto the unital Jordan $C(A)$-subalgebra of $Q(A)$ generated by $J$, which is the identity map when restricted to $J$.

THEOREM 4.4. Let $A$ be a prime associative algebra with involution * and let $J$ be a Jordan subalgebra of $H(Q(A), *)$ containing $H(A, *)$ as an ideal. Then there is an isomorphism from $C(J)$ onto $H(C(A), *)$ that extends to an isomorphism from the unital central closure of $J$ onto the unital Jordan $H(C(A), *)$-subalgebra of $Q(A)$ generated by $J$, which is the identity map when restricted to $J$.

THEOREM 4.5. Let $J$ be a prime nondegenerate Jordan algebra with $\operatorname{deg}(J) \geq 12$. Then one of the following assertions holds:

(i) There exists a prime associative algebra $A$ such that $J$ is a 5 -free Jordan subalgebra of $Q(A)$ and $A$ is an ideal of $J$.

(ii) There exists a prime associative algebra $A$ with involution $*$ such that $J$ is a Jordan subalgebra of $H(Q(A), *)$ containing $H(A, *)$ as an ideal and $J$ is a 5 -free subset of $Q(A)$. 
Proof. We first remark that $J$ is as in cases (3) or (4) of Zelmanov's theorem. Namely, otherwise we would have $\operatorname{deg}(J) \leq 3$ (cf. [29, Examples D and E, p. 232]).

Suppose that there exists a prime associative algebra $A$ such that $J$ is a Jordan subalgebra of $Q(A)$ containing $A$ as an ideal. Our next objective is to show that

$$
\operatorname{deg}(A)=\operatorname{deg}(J) .
$$

It should be pointed out that, on account of Theorem 4.3, we have

$$
\operatorname{deg}(A)=\operatorname{deg}_{Q(A)}(A) \leq \operatorname{deg}_{Q(A)}(J)=\operatorname{deg}(J) \leq \operatorname{deg}(Q(A)) .
$$

In the case when $\operatorname{deg}(A)=\infty$, equality (6) obviously holds true. We proceed to the case when $\operatorname{deg}(A)=n<\infty$. By [10, Corollary 2.3.8] we have

$$
\sum_{\sigma \in S_{n+1}}(-1)^{\sigma} a^{\sigma(0)} b_{1} a^{\sigma(1)} b_{2} \cdots b_{n} a^{\sigma(n)}=0
$$

for all $a, b_{1}, b_{2}, \ldots, b_{n} \in A$, where $S_{n+1}$ is the symmetric group of degree $n+1$ and $(-1)^{\sigma}$ is the sign of the permutation $\sigma$. Consider

$$
\begin{aligned}
\phi\left(\mathbf{x}, \mathbf{y}_{1}, \mathbf{y}_{2}, \ldots, \mathbf{y}_{n}\right) & =\sum_{\sigma \in S_{n+1}}(-1)^{\sigma} \mathbf{x}^{\sigma(0)} \mathbf{y}_{1} \mathbf{x}^{\sigma(1)} \mathbf{y}_{2} \cdots \mathbf{y}_{n} \mathbf{x}^{\sigma(n)} \\
& \in C(A)\left\langle\mathbf{x}, \mathbf{y}_{1}, \mathbf{y}_{2}, \ldots, \mathbf{y}_{n}\right\rangle .
\end{aligned}
$$

Since $\phi$ is a GPI on $A$, [10, Theorem 6.4.4] shows that it is a GPI on $Q(A)$. Accordingly, by [10, Corollary 2.3.8], $\operatorname{deg}(Q(A)) \leq n$. Using (7) we now see that (6) indeed holds true. From [7, Theorems 2.4 and 2.8] it follows that $J$ is a 5 -free subset of $Q(A)$.

We now turn to the case when there exists a prime associative algebra $A$ with involution $*$ such that $J$ is a Jordan subalgebra of $H(Q(A), *)$ containing $H(A, *)$ as an ideal. On account of Theorem 4.4, we have

$$
\begin{aligned}
\operatorname{deg}(H(A, *)) & =\operatorname{deg}_{H(Q(A), *)}(H(A, *)) \\
& \leq \operatorname{deg}_{H(Q(A), *)}(J)=\operatorname{deg}(J) \leq \operatorname{deg}(H(Q(A), *)) .
\end{aligned}
$$

We claim that $\operatorname{deg}_{H(Q(A), *)}(h) \leq \operatorname{deg}_{Q(A)}(h)$ for each $h \in H(Q(A), *)$. Let $h \in H(Q(A), *)$ with $\operatorname{deg}_{Q(A)}(h)=n<\infty$. There exist $c_{0}, c_{1}, \ldots, c_{n} \in C(A)$ with $c_{n} \neq 0$ such that $\sum_{i=0}^{n} c_{i} h^{i}=0$, and we can certainly assume that $c_{n}=1$. Thus $\sum_{i=0}^{n} c_{i}^{*} h^{i}=0$ and therefore $\sum_{i=0}^{n-1} \frac{1}{2}\left(c_{i}+c_{i}^{*}\right) h^{i}+h^{n}=0$. Since $\frac{1}{2}\left(c_{i}+c_{i}^{*}\right) \in H(C(A), *)=C(H(A, *))$, the latter equality shows that $\operatorname{deg}_{H(Q(A), *)}(h) \leq n$, as claimed. Therefore

$$
\operatorname{deg}_{H(Q(A), *)}(H(Q(A), *)) \leq \operatorname{deg}_{Q(A)}(H(Q(A), *)) \leq \operatorname{deg}_{Q(A)}(Q(A)) .
$$

As in the proof of the previous case we have $\operatorname{deg}(Q(A))=\operatorname{deg}(A)$. This fact together with $(8)$ gives $\operatorname{deg}(A) \geq 12$. Now [7, Theorems 2.4 and 2.8] show that $J$ is a 5 -free subset of $Q(A)$. 
We are now in a position to prove Theorem 1.2.

Proof of Theorem 1.2. Let $C$ be the extended centroid of $J$, let $H_{C}$ and $J_{C}$ denote the respective scalar extensions of $H$ and $J$ over $C$, and let $\Phi_{C}: H_{C} \rightarrow J_{C}$ be the Lie triple epimorphism induced by $\Phi$. Keeping in mind that the central closure $Q_{0}(J)$ of $J$ is a quotient of $J_{C}$, and considering the quotient map $\varrho: J_{C} \rightarrow Q_{0}(J)$, it follows that $\Phi_{0}:=\varrho \Phi_{C}$ is a Lie triple epimorphism from $H_{C}$ onto $Q_{0}(J)$ which extends $\Phi$. Consider the unital central closure $\widehat{J}$ of $J$, choose a $C$-subspace $J_{0}$ of $Q_{0}(J)$ such that $\widehat{J}=J_{0} \oplus C$, and denote by $\pi_{0}$ the corresponding projection from $Q_{0}(J)$ onto $J_{0}$. Note that $J_{0}=Q_{0}(J)$ whenever $Q_{0}(J)$ lacks unity. In any case, it follows that $q-\pi_{0}(q) \in C$ for each $q \in Q_{0}(J)$.

Let $\widetilde{H}$ denote the Jordan algebra obtained as the direct product of $H_{C}$ and $C$. Let us consider the map $\alpha: \widetilde{H} \rightarrow \widehat{J}$ defined by $\alpha(a, c)=\pi_{0} \Phi_{0}(a)+c$. It is clear that $\alpha$ is a surjective $C$-linear map. Moreover, it is easy to see that $[\alpha(x), \alpha(y), \alpha(z)] \in Q_{0}(J)$ and $\pi_{0}([\alpha(x), \alpha(y), \alpha(z)])=\alpha([x, y, z])$ for all $x, y, z \in \widetilde{H}$. Thus, we have

$$
\alpha([x, y, z])-[\alpha(x), \alpha(y), \alpha(z)] \in C
$$

for all $x, y, z \in \widetilde{H}$. We now define the map $B: \widetilde{H} \times \widetilde{H} \rightarrow \widehat{J}$ by $B(x, y)=$ $\alpha(x \circ y)$. By (9) and the linearization of the Jordan identity, we have

$$
[B(x, y), \alpha(z), \alpha(t)]+[B(t, x), \alpha(z), \alpha(y)]+[B(y, t), \alpha(z), \alpha(x)] \in C
$$

for all $x, y, z, t \in \widetilde{H}$. We apply Theorem 4.5 and from now on we consider the prime associative algebra $A$ given by that result.

By Theorem 4.2, there exist $\lambda_{1}, \lambda_{2} \in C(A)$, linear maps $\mu_{1}, \mu_{2}: \widetilde{H} \rightarrow$ $C(A)$, and a bilinear map $\nu: \widetilde{H} \times \widetilde{H} \rightarrow C(A)$ such that

$$
B(x, y)=\lambda_{1} \alpha(x) \alpha(y)+\lambda_{2} \alpha(y) \alpha(x)+\mu_{1}(x) \alpha(y)+\mu_{2}(y) \alpha(x)+\nu(x, y)
$$

for all $x, y \in \widetilde{H}$. Since $B$ is symmetric, it follows that

$$
\begin{aligned}
\left(\lambda_{1}-\lambda_{2}\right) \alpha(x) \alpha(y)+( & \left.\lambda_{2}-\lambda_{1}\right) \alpha(y) \alpha(x)+\left(\mu_{1}(x)-\mu_{2}(x)\right) \alpha(y) \\
& +\left(\mu_{2}(y)-\mu_{1}(y)\right) \alpha(x)+\nu(x, y)-\nu(y, x)=0
\end{aligned}
$$

for all $x, y \in \widetilde{H}$. Then, taking into account Theorem 4.1, we conclude that

$$
\lambda_{1}=\lambda_{2}, \quad \mu_{1}=\mu_{2}, \quad \nu(x, y)=\nu(y, x)
$$

for all $x, y \in \widetilde{H}$. Moreover, in the case when $A$ has an involution and $J$ is a Jordan subalgebra of $H(Q(A), *)$ containing $H(A, *)$ as an ideal, both $B$ and $\alpha$ are $H(Q(A), *)$-valued, and hence we have

$$
\begin{aligned}
\left(\lambda_{1}-\lambda_{1}^{*}\right) \alpha(x) \alpha(y)+ & \left(\lambda_{1}-\lambda_{1}^{*}\right) \alpha(y) \alpha(x)+\left(\mu_{1}(x)-\mu_{1}(x)^{*}\right) \alpha(y) \\
& +\left(\mu_{1}(y)-\mu_{1}(y)^{*}\right) \alpha(x)+\nu(x, y)-\nu(x, y)^{*}=0
\end{aligned}
$$


for all $x, y \in \widetilde{H}$. Applying Theorem 4.1 again, we see that $\lambda_{1} \in C$, and that the maps $\mu_{1}$ and $\nu$ are $C$-valued.

Summarizing, there exist $\lambda=2 \lambda_{1} \in C$, a linear map $\mu=\mu_{1}: \widetilde{H} \rightarrow C$, and a symmetric bilinear map $\nu: \widetilde{H} \times \widetilde{H} \rightarrow C$ such that

$$
\alpha(x \circ y)=\lambda \alpha(x) \circ \alpha(y)+\mu(x) \alpha(y)+\mu(y) \alpha(x)+\nu(x, y)
$$

for all $x, y \in \widetilde{H}$. It follows that

$$
\begin{aligned}
\alpha((x \circ y) \circ z)= & \lambda^{2}(\alpha(x) \circ \alpha(y)) \circ \alpha(z)+\lambda \mu(y) \alpha(x) \circ \alpha(z) \\
& +\lambda \mu(x) \alpha(y) \circ \alpha(z)+\lambda \mu(z) \alpha(x) \circ \alpha(y)+\mu(y) \mu(z) \alpha(x) \\
& +\mu(x) \mu(z) \alpha(y)+(\lambda \nu(x, y)+\mu(x \circ y)) \alpha(z)+\nu(x, y) \mu(z) \\
& +\nu(x \circ y, z) .
\end{aligned}
$$

Analogously

$$
\begin{aligned}
\alpha(x \circ(y \circ z))= & \lambda^{2} \alpha(x) \circ(\alpha(y) \circ \alpha(z))+\lambda \mu(z) \alpha(x) \circ \alpha(y) \\
& +\lambda \mu(y) \alpha(x) \circ \alpha(z)+\lambda \mu(x) \alpha(y) \circ \alpha(z) \\
& +(\lambda \nu(y, z)+\mu(y \circ z)) \alpha(x)+\mu(x) \mu(z) \alpha(y) \\
& +\mu(x) \mu(y) \alpha(z)+\mu(x) \nu(y, z)+\nu(x, y \circ z) .
\end{aligned}
$$

As a consequence,

$$
\begin{aligned}
\alpha([x, y, z])= & \lambda^{2}[\alpha(x), \alpha(y), \alpha(z)]+(\mu(y) \mu(z)-\lambda \nu(y, z)-\mu(y \circ z)) \alpha(x) \\
& +(\lambda \nu(x, y)+\mu(x \circ y)-\mu(x) \mu(y)) \alpha(z)+\nu(x, y) \mu(z) \\
& +\nu(x \circ y, z)-\mu(x) \nu(y, z)-\nu(x, y \circ z) .
\end{aligned}
$$

Comparing this expression with (9), and applying Theorem 4.1, we get

$$
\lambda^{2}=1, \quad \lambda \nu(x, y)+\mu(x \circ y)-\mu(x) \mu(y)=0 .
$$

Now, we consider the map $\beta: \widetilde{H} \rightarrow \widehat{J}$ defined by $\beta(x)=\lambda \alpha(x)+\mu(x)$ for each $x \in \widetilde{H}$. It follows easily from (10) and (11) that $\beta$ is a homomorphism as well. Therefore, the map $\Psi: H \rightarrow \widehat{J}$ defined by $\Psi(a)=\beta(a, 0)$ for each $a \in H$ is a homomorphism as well. From the definitions of $\alpha, \Psi$, and $\beta$ it follows that

$$
\begin{aligned}
\pi_{0} \Phi(a) & =\pi_{0} \Phi_{0}(a)=\alpha(a, 0)=\lambda^{-1} \beta(a, 0)-\lambda^{-1} \mu(a, 0) \\
& =\lambda^{-1} \Psi(a)-\lambda^{-1} \mu(a, 0)
\end{aligned}
$$

for each $a \in H$. From this and the fact that $\lambda= \pm 1$ (by (11)), we deduce that $\Phi(a)-\lambda \Psi(a) \in C$. Finally, define $\tau: H \rightarrow C$ by the equality $\Phi(a)=$ $\lambda \Psi(a)+\tau(a)$ for each $a \in H$. It is clear that $\tau$ is a linear map, and it is easy to check that $\tau([H, H, H])=0$.

5. Continuity of Lie triple epimorphisms. Throughout this last section we use two results coming from automatic continuity theory. The first one is by B. Aupetit [3, Theorem 2]. 
TheOREm 5.1. Let $H$ and $J$ be Jordan-Banach algebras and let $\Phi$ be an epimorphism from $H$ onto $J$. If $J$ is semisimple then $\Phi$ is continuous.

The second result is taken from [37, Lemma 3.5]. In order to state it we need to introduce some terminology. By a nonassociative Banach algebra we mean a nonassociative complex algebra $B$ whose underlying space is a Banach space with respect to a norm $\|\cdot\|$ satisfying $\|a \cdot b\| \leq \gamma\|a\|\|b\|$ for all $a, b \in B$ and some $\gamma>0$.

Lemma 5.2. Let $B$ and $C$ be nonassociative Banach algebras and let $\Phi$ be an epimorphism from $B$ onto $C$. Then the multiplication operator $x \mapsto a \cdot x$ from $C$ into itself is quasi-nilpotent for each $a \in \mathfrak{S}(\Phi)$.

Let $J$ be a Jordan-Banach algebra. For all $a, b \in J$ we define the continuous linear operator $L(a, b): J \rightarrow J$ by

$$
L(a, b)(x)=[a, b, x]
$$

for each $x \in J$.

Lemma 5.3. Let $H$ and $J$ be Jordan-Banach algebras and let $\Phi: H \rightarrow J$ be a Lie triple epimorphism. Then $\mathfrak{S}(\Phi)$ is a closed Lie triple ideal of $J$ and $L(a, b)$ is a quasi-nilpotent operator on $J$ for all $a \in \mathfrak{S}(\Phi)$ and $b \in J$.

Proof. We begin by proving that $\mathfrak{S}(\Phi)$ is a Lie triple ideal of $J$. Let $a \in$ $\mathfrak{S}(\Phi)$ and $b, c \in J$. Then there exists a sequence $\left(x_{n}\right)$ in $H$ with $\lim x_{n}=0$ and $\lim \Phi\left(x_{n}\right)=a$ and there exist $y, z \in H$ with $\Phi(y)=b$ and $\Phi(z)=c$. We have $\lim \left[x_{n}, y, z\right]=0$ and

$$
\lim \Phi\left[x_{n}, y, z\right]=\lim \left[\Phi\left(x_{n}\right), b, c\right]=[a, b, c],
$$

which shows that $[a, b, c] \in \mathfrak{S}(\Phi)$.

Fix $b \in J$ and let $v \in H$ be such that $\Phi(v)=b$. We now turn both $H$ and $J$ into nonassociative Banach algebras by defining $x \cdot{ }_{v} y=[x, v, y]$ and $a{ }_{b} c=[a, b, c]$ for all $x, y \in H$ and $a, c \in J$. Of course, $\Phi\left(x \cdot{ }_{v} y\right)=\Phi(x) \cdot{ }_{b} \Phi(y)$ and Lemma 5.2 now shows that the operator $c \mapsto a{ }_{b} c$ from $J$ into itself is quasi-nilpotent for each $a \in \mathfrak{S}(\Phi)$. Note that the latter operator is nothing other than the operator $L(a, b)$ on $J$.

Lemma 5.4. Let $H$ and $J$ be Jordan-Banach algebras and let $\Phi: H \rightarrow J$ be a Lie triple epimorphism. Assume that $J$ is primitive and either finitedimensional or quadratic. Then $\mathfrak{S}(\Phi) \subseteq Z(J)$.

Proof. To obtain a contradiction, suppose that $\mathfrak{S}(\Phi) \nsubseteq Z(J)$. Then $J$ is not associative, so that, by the structure theory [29, Chap. V], $J$ is isomorphic to either $H_{3}(\mathbb{O}), M_{n}(\mathbb{C})^{+}$with $n \geq 2, H\left(M_{n}(\mathbb{C}),{ }^{t}\right)$ with $n \geq 2$ (where ${ }^{\mathrm{t}}$ stands for the transpose involution), $H\left(M_{2 n}(\mathbb{C}),{ }^{\mathrm{s}}\right)$ with $n \geq 2$ (where ${ }^{\mathrm{s}}$ stands for the symplectic involution), or $J(X, f)$ with $\operatorname{dim} X \geq 2$. Therefore, in every case, $J$ contains a nondegenerate Jordan subalgebra 
which is quadratic and of dimension $\geq 3$. Thus, by Lemma 3.3, there exist $a, b, c \in[J, J, J] \backslash\{0\}$ such that $L(a, b)(c)=c$. Since $J$ is simple, according to Lemma 5.3 and Theorem 1.1, $\mathfrak{S}(\Phi)$ is a Lie triple ideal of $J$ containing $[J, J, J]$ and the operator $L(a, b)$ on $J$ is quasi-nilpotent, which contradicts $L(a, b)(c)=c$.

Lemma 5.5. Let $H$ be a Jordan-Banach algebra, let $J$ be a primitive Jordan-Banach algebra, and let $\Phi: H \rightarrow J$ be a Lie triple epimorphism. Suppose that $\operatorname{deg}(J) \geq 12$. Then $\mathfrak{S}(\Phi) \subseteq Z(J)$.

Proof. By [35, Theorem 12], $J$ is centrally closed, and so the norm of $J$ extends in an obvious way to a complete algebra norm on $\widehat{J}$. On the other hand, by Theorem 1.2, there exist a homomorphism $\Psi: H \rightarrow \widehat{J}$ and a linear map $\tau: H \rightarrow \mathbb{C}$ satisfying $\tau([H, H, H])=0$ such that $\Phi= \pm \Psi+\tau$. Given $y \in \widehat{J}$, we write $y=b+z \mathbf{1}$ with $b \in J$ and $z \in \mathbb{C}$ and we choose $a \in H$ such that $\Phi(a)=b$. We see at once that $y= \pm \Psi(a)+\tau(a) \mathbf{1}+z \mathbf{1}$ and hence $y \in \Psi(H)+\mathbb{C} 1$. Therefore $\widehat{J}=\Psi(H)+\mathbb{C} 1$. It follows that $\Psi(H)$ is an ideal of $\widehat{J}$ with codimension 0 or 1 . Thus, either $\Psi(H)=\widehat{J}$ or $\Psi(H)$ is a maximal ideal of $\widehat{J}$, and consequently closed. Therefore, in any case, $\Psi(H)$ is a Jordan-Banach algebra. Clearly $J \neq \mathbb{C}$ and hence $\Psi(H) \neq 0$.

Since $\widehat{J}$ is primitive (see [28]) and $\Psi(H) \neq 0,[2$, Theorem 1.1] now shows that $\Psi(H)$ is a primitive Jordan-Banach algebra. Thus, $\Psi$ is an epimorphism from the Jordan-Banach algebra $H$ onto the primitive Jordan-Banach algebra $\Psi(H)$, and consequently, by Theorem 5.1, $\Psi$ is continuous. Finally, given $b \in \mathfrak{S}(\Phi)$, we can choose a null sequence $\left(a_{n}\right)$ in $H$ such that $\left(\Phi\left(a_{n}\right)\right)$ converges to $b$, so that the equality $\Phi\left(a_{n}\right)= \pm \Psi\left(a_{n}\right)+\tau\left(a_{n}\right) \mathbf{1}$ for each $n$ implies that $b \in \overline{\mathbb{C} 1}$. Since $\mathbb{C} \mathbf{1}$ is finite-dimensional, and so closed in $\widehat{J}$, we conclude that $b \in \mathbb{C} \mathbf{1} \cap J \subseteq Z(J)$.

Proof of Theorem 1.3. It suffices to prove that $[\mathfrak{S}(\Phi), J, J] \subseteq P$ for each primitive ideal $P$ of $J$. Let $P$ be a primitive ideal of $J$. It is known that $P$ is closed in $J$ ([23, Lemma 6.5]). Let us consider the quotient map $\pi_{P}$ : $J \rightarrow J / P$. Then $J / P$ is a primitive Jordan-Banach algebra and $\pi_{P} \Phi$ is a Lie triple epimorphism from $H$ onto $J / P$. We claim that $\mathfrak{S}\left(\pi_{P} \Phi\right) \subseteq Z(J / P)$. On account of Lemma 5.5, this is certainly true in the case when $\operatorname{deg}(J / P) \geq 12$. In the case $\operatorname{deg}(J / P)<12$ the spectrum of every element in $J / P$ is obviously finite and from [11] it may be concluded that $J / P$ is either finite-dimensional or quadratic. Lemma 5.4 concludes the proof of the claim.

Since $\pi_{P}$ is continuous, it is a standard fact that

$$
\mathfrak{S}\left(\pi_{P} \Phi\right)=\overline{\pi_{P}(\mathfrak{S}(\Phi))}
$$

Thus we proved that $\pi_{P}(\mathfrak{S}(\Phi)) \subseteq Z(J / P)$, which entails that $[\mathfrak{S}(\Phi), J, J]$ $\subseteq P$. 
REMARK 5.6.

(1) Let $A$ and $B$ be Banach algebras and let $\Phi: A \rightarrow B$ be a Lie epimorphism. Then the symmetrized algebras $A^{+}$and $B^{+}$are JordanBanach algebras and $\Phi$ becomes a Lie triple epimorphism from $A^{+}$ onto $B^{+}$. According to Theorem 1.3, $\mathfrak{S}(\Phi) \subseteq \mathcal{Z}\left(B^{+}\right)$. Since

$$
\operatorname{Rad}\left(B^{+}\right)=\operatorname{Rad}(B)
$$

(see [33]) and

$$
Z\left(B^{+} / \operatorname{Rad}\left(B^{+}\right)\right)=Z(B / \operatorname{Rad}(B))
$$

(see [21]), we have $\mathcal{Z}\left(B^{+}\right)=\mathcal{Z}(B)$, and so [4, Theorem] follows.

(2) By means of the standard procedure of complexification we can arrive at the same conclusion of Theorem 1.3 without the requirement of $H$ and $J$ being complex.

\section{References}

[1] J. A. Anquela, T. Cortés, and E. García, Herstein's theorems and simplicity of hermitian Jordan systems, J. Algebra 246 (2001), 193-214.

[2] J. A. Anquela, F. Montaner, and T. Cortés, On primitive Jordan algebras, ibid. 163 (1994), 663-674.

[3] B. Aupetit, The uniqueness of the complete norm topology in Banach algebras and Banach Jordan algebras, J. Funct. Anal. 47 (1982), 1-6.

[4] B. Aupetit and M. Mathieu, The continuity of Lie homomorphisms, Studia Math. 138 (2000), 193-199.

[5] W. E. Baxter and W. S. Martindale III, Jordan homomorphisms of semiprime rings, J. Algebra 56 (1979), 457-471.

[6] - - - Central closure of semiprime nonassociative rings, Comm. Algebra 7 (1979), 1103-1132.

[7] K. I. Beidar and M. A. Chebotar, On functional identities and d-free subsets of rings. I, Comm. Algebra 28 (2000), 3925-3951.

[8] -, -, On functional identities and d-free subsets of rings. II, ibid. 28 (2000), 3953-3972.

[9] -, - On surjective Lie homomorphisms onto Lie ideals of prime rings, ibid. 29 (2001), 4775-4793.

[10] K. I. Beidar, W. S. Martindale III, and A. V. Mikhalev, Rings with Generalized Identities, Marcel Dekker, New York, 1996.

[11] M. Benslimane et A. M. Kaidi, Structure des algèbres de Jordan-Banach non commutatives complexes régulières ou semi-simples à spectre fini, J. Algebra 113 (1988), 201-206.

[12] M. I. Berenguer and A. R. Villena, Continuity of Lie isomorphisms of Banach algebras, Bull. London Math. Soc. 31 (1999), 6-10.

[13] J. Bergen, I. N. Herstein, and J. W. Kerr, Lie ideals and derivations of prime rings, J. Algebra 71 (1981), 259-267.

[14] M. Brešar, Centralizing mappings and derivations in prime rings, ibid. 156 (1993), $385-394$. 
[15] M. Brešar, Commuting traces and biadditive mappings, commutativity-preserving mappings and Lie mappings, Trans. Amer. Math. Soc. 335 (1993), 525-546.

[16] -, Functional identities: a survey, in: Contemp. Math. 259, Amer. Math. Soc., Providence, RI, 2000, 93-109.

[17] M. Brešar, M. Cabrera, and A. R. Villena, Functional identities in Jordan algebras: associating maps, Comm. Algebra 30 (2002), 5241-5252.

[18] M. Brešar, D. Eremita, and A. R. Villena, Functional identities in Jordan algebras: associating traces and Lie triple isomorphisms, ibid. 31 (2003), 1207-1234.

[19] M. Cabrera, A. Moreno, and Á. Rodríguez, Normed versions of the Zel'manov prime theorem: positive results and limits, in: Operator Theory, Operator Algebras and Related Topics (Timişoara, 1996), A. Gheondea, R. N. Gologan, and D. Timotin (eds.), The Theta Foundation, Bucharest, 1997, 65-77.

[20] - , - - Z Zel'manov's theorem for primitive Jordan-Banach algebras, J. London Math. Soc. 57 (1998), 231-244.

[21] M. Cabrera and A. Rodríguez, A characterization of the centre of a nondegenerate Jordan algebra, Comm. Algebra 21 (1993), 359-369.

[22] T. S. Erickson, W. S. Martindale III, and J. M. Osborn, Prime nonassociative algebras, Pacific J. Math. 60 (1975), 49-63.

[23] A. Fernández, Modular annihilator Jordan algebras, Comm. Algebra 13 (1985), $2597-2613$.

[24] I. N. Herstein, Lie and Jordan structures in simple, associative rings, Bull. Amer. Math. Soc. 67 (1961), 517-531.

[25] —, Topics in Ring Theory, The University of Chicago Press, Chicago, 1969.

[26] - , On the Lie structure of an associative ring, J. Algebra 14 (1970), 561-571.

[27] —, Rings with Involution, The University of Chicago Press, Chicago, 1976.

[28] L. Hogben and K. McCrimmon, Maximal modular inner ideals and the Jacobson radical of a Jordan algebra, J. Algebra 68 (1981), 155-169.

[29] N. Jacobson, Structure and Representations of Jordan Algebras, Amer. Math. Soc. Colloq. Publ. 39, Amer. Math. Soc., Providence, RI, 1968.

[30] P.-H. Lee and T.-K. Lee, Linear identities and commuting maps in rings with involution, Comm. Algebra 25 (1997), 2881-2895.

[31] W. S. Martindale III and C. R. Miers, Herstein's Lie theory revisited, J. Algebra 98 (1986), 14-37.

[32] K. McCrimmon, On Herstein's theorems relating Jordan and associative algebras, ibid. 13 (1969), 382-392.

[33] —, The radical of a Jordan algebra, Proc. Nat. Acad. Sci. U.S.A. 59 (1969), 671-678.

[34] K. McCrimmon and E. Zelmanov, The structure of strongly prime quadratic Jordan algebras, Adv. Math. 69 (1988), 133-222.

[35] Á. Rodríguez, Primitive nonassociative normed algebras and extended centroid, in: Nonassociative Algebraic Models, S. González and H. C. Myung (eds.), Nova Science Publ., New York, 1992, 233-243.

[36] V. G. Skosyrskiǔ, Primitive Jordan algebras, Algebra i Logika 31 (1992), 174-188 (in Russian); English transl.: Algebra and Logic 31 (1992), 110-120.

[37] A. R. Villena, Automatic continuity in associative and nonassociative context, Irish Math. Soc. Bull. 46 (2001), 43-76.

[38] E. I. Zelmanov, Prime Jordan algebras, Algebra i Logika 18 (1979), 162-175 (in Russian); English transl.: Algebra and Logic 18 (1979), 103-111.

[39] —, Prime Jordan algebras II, Sibirsk. Mat. Zh. 24 (1983), no. 1, 89-104 (in Russian); English transl.: Siberian Math. J. 24 (1983), no. 1, 73-85. 
[40] E. I. Zelmanov, Characterization of the McCrimmon radical, Sibirsk. Mat. Zh. 25 (1984), no. 5, 190-192 (in Russian).

[41] K. A. Zhevlakov, A. M. Slinko, I. P. Shestakov, and A. I. Shirshov, Rings That Are Nearly Associative, Academic Press, New York, 1982.

Department of Mathematics

University of Maribor

PEF, Koroška 160

2000 Maribor, Slovenia

E-mail: bresar@uni-mb.si

Institute of Mathematics, Physics, and Mechanics

Jadranska 19

1000 Ljubljana, Slovenia

E-mail: maja.fosner@uni-mb.si
Departamento de Análisis Matemático Facultad de Ciencias Universidad de Granada 18071 Granada, Spain E-mail: cabrera@ugr.es avillena@ugr.es 